\title{
Fixed Point Theorems for a Class of Weakly C-Contractive Mappings in a Setting of 2-Banach Space
}

\author{
M. Saha ${ }^{1}$ and Anamika Ganguly ${ }^{2}$ \\ ${ }^{1}$ Department of Mathematics, The University of Burdwan, Burdwan, West Bengal, 713104, India \\ ${ }^{2}$ Burdwan Railway Balika Vidyapith High School, Khalasipara, Burdwan, West Bengal, 713101, India \\ Correspondence should be addressed to Anamika Ganguly; anamika.simi@gmail.com
}

Received 26 September 2012; Accepted 1 December 2012

Academic Editor: Baoding Liu

Copyright (c) 2013 M. Saha and A. Ganguly. This is an open access article distributed under the Creative Commons Attribution License, which permits unrestricted use, distribution, and reproduction in any medium, provided the original work is properly cited.

In this paper, the authors have proved some existence theorems of fixed points for a class of weakly C-contractive mappings in a setting of 2-Banach space. The authors have supported the results with the help of suitable examples.

\section{Introduction}

The theory of 2-Banach spaces was investigated by Gähler in [1] and Iseki in [2] who proved some fixed point theorems in such spaces and these new spaces have subsequently been studied by many authors. For example, we refer to $[3,4]$ where authors have dealt with mappings that are of contractive nature. Our findings as presented here have added further findings in fixed point theory in a 2-Banach space by working with a class of mappings so general that take care of those as found in $[5,6]$.

We now state some definitions before presenting our main results.

\section{Preliminaries}

Definition 1 . Let $X$ be a real linear space and $\|\cdot, \cdot\|$ a nonnegative real valued function defined on $X$ satisfying the following conditions:

(i) $\|x, y\|=0$ if and only if $x$ and $y$ are linearly dependent,

(ii) $\|x, y\|=\|y, x\|$ for all $x, y \in X$,

(iii) $\|x, \alpha y\|=|\alpha|\|x, y\|, \alpha$ being real for all $x, y \in X$,

(iv) $\|x, y+z\| \leq\|x, y\|+\|x, z\|$ for all $x, y, z \in X$.
Then $\|\cdot, \cdot\|$ is called a 2 -norm and the pair $(X,\|\cdot, \cdot\|)$ is called a linear 2-normed space. So a 2-norm $\|x, y\|$ always satisfies $\|x, y+\alpha x\|=\|x, y\|$ for all $x, y \in X$ and all scalars $\alpha$.

Definition 2. A sequence $\left\{x_{n}\right\}$ in a linear 2-normed space $(X,\|\cdot, \cdot\|)$ is said to be a Cauchy sequence if $\lim _{n, m \rightarrow \infty} \| x_{n}-$ $x_{m}, a \|=0$ for all $a$ in $X$.

Definition 3. A sequence $\left\{x_{n}\right\}$ in a linear 2-normed space $(X,\|\cdot, \cdot\|)$ is said to be convergent in $X$ if there exists a point $x$ in $X$ such that $\lim _{n \rightarrow \infty}\left\|x_{n}-x, a\right\|=0$ for all $a$ in $X$. If $\left\{x_{n}\right\}$ converges to $x$, we write $x_{n} \rightarrow x$ as $n \rightarrow \infty$.

Definition 4. A linear 2-normed space $X$ is said to be complete if every Cauchy sequence is convergent to an element of $X$. A complete linear 2-normed space $X$ is called a 2-Banach space.

The main purpose of our paper is to find out the existence of fixed point for a class of weakly $\mathrm{C}$-contractive results due to Choudhury [7] in a setting of 2-Banach space. We have also established a common fixed point theorem for such type of mappings with a supporting example. Some allied theorems have also been presented here.

\section{Main Results}

Definition 5. A mapping $T:(X,\|\cdot, \cdot\|) \rightarrow(X,\|\cdot, \cdot\|)$ is said to be weakly $C$-contractive or a weak $C$-contraction if for all $x, y, a$ in $X$, 


$$
\begin{aligned}
\|T(x)-T(y), a\| \leq & \frac{1}{2}[\|x-T(y), a\|+\|y-T(x), a\|] \\
& -\phi[\|x-T(y), a\|,\|y-T(x), a\|],
\end{aligned}
$$

where $\phi:[0, \infty)^{2} \rightarrow[0, \infty)$ is a continuous mapping such that $\phi(x, y)=0$ if and only if $x=y=0$.

Theorem 6. Let $(X,\|\cdot, \cdot\|)$ be a 2-Banach space and let $T$ : $X \rightarrow X$ be a weak $C$-contraction. Then $T$ has a unique fixed point in $X$.

Proof. Let $x_{0} \in X$. Let $x_{n+1}=T\left(x_{n}\right), n=1,2, \ldots$

If $x_{n}=x_{n+1}=T\left(x_{n}\right)$, then $T$ has a fixed point $x_{n}$.

Assume that $x_{n} \neq x_{n+1}$. Now for any $a \in X$,

$$
\begin{aligned}
\left\|x_{n}-x_{n+1}, a\right\|= & \left\|T\left(x_{n-1}\right)-T\left(x_{n}\right), a\right\| \\
\leq & \frac{1}{2}\left[\left\|x_{n-1}-T\left(x_{n}\right), a\right\|+\left\|x_{n}-T\left(x_{n-1}\right), a\right\|\right] \\
& -\phi\left[\left\|x_{n-1}-T\left(x_{n}\right), a\right\|,\left\|x_{n}-T\left(x_{n-1}\right), a\right\|\right] \\
\leq & \frac{1}{2}\left[\left\|x_{n-1}-x_{n}, a\right\|+\left\|x_{n}-x_{n+1}, a\right\|\right] \\
& -\phi\left[\left\|x_{n-1}-x_{n+1}, a\right\|, 0\right] .
\end{aligned}
$$

Therefore,

$$
\left\|x_{n}-x_{n+1}, a\right\| \leq\left\|x_{n-1}-x_{n}, a\right\| .
$$

So $\left\{\left\|x_{n}-x_{n+1}, a\right\|\right\}$ is a monotone decreasing sequence of real numbers and hence $\left\{\left\|x_{n}-x_{n+1}, a\right\|\right\}$ is convergent and let $\| x_{n}-$ $x_{n+1}, a \| \rightarrow r$ (say) as $n \rightarrow \infty$

We now show that $r=0$. If not, then

$$
\begin{aligned}
\left\|x_{n}-x_{n+1}, a\right\| & =\left\|T\left(x_{n-1}\right)-T\left(x_{n}\right), a\right\| \\
& \leq \frac{1}{2}\left\|x_{n-1}-x_{n+1}, a\right\| \\
& \leq \frac{1}{2}\left[\left\|x_{n-1}-x_{n}, a\right\|+\left\|x_{n}-x_{n+1}, a\right\|\right] .
\end{aligned}
$$

Taking $n \rightarrow \infty$, we get

$$
\begin{aligned}
r \leq & \frac{1}{2} \lim _{n \rightarrow \infty}\left\|x_{n-1}-x_{n+1}, a\right\| \\
& +\frac{1}{2} \lim _{n \rightarrow \infty}\left\|x_{n}-x_{n+1}, a\right\| \leq \frac{1}{2} r+\frac{1}{2} r,
\end{aligned}
$$

implying that

$$
\lim _{n \rightarrow \infty}\left\|x_{n-1}-x_{n+1}, a\right\|=2 r .
$$

Also from (2) and using continuity of $\phi$, we get $r \leq$ $r-\phi[2 r, 0]$ showing that $\phi[2 r, 0] \leq 0$, a contradiction, unless

$$
r=0 \text {. So, }\left\|x_{n}-x_{n+1}, a\right\| \rightarrow 0 \text { as } n \rightarrow \infty \text {, that is }
$$$$
\lim _{n \rightarrow \infty}\left\|x_{n}-x_{n+1}, a\right\|=0
$$

We shall now show that $\left\{x_{n}\right\}$ is Cauchy sequence in $X$.

If not, then there exists a $\varepsilon>0$ and for each positive integer $k$, there exist integers $n(k)$ and $m(k)$ with $k<m(k)<$ $n(k)$ such that $\left\|x_{m(k)}-x_{n(k)}, a\right\| \geq \varepsilon$ and $\left\|x_{m(k)}-x_{n(k)-1}, a\right\|<\varepsilon$. Then

$$
\begin{aligned}
\varepsilon \leq & \left\|x_{m(k)}-x_{n(k)}, a\right\|=\left\|T\left(x_{m(k)-1}\right)-T\left(x_{n(k)-1}\right), a\right\| \\
\leq & \frac{1}{2}\left[\left\|x_{m(k)-1}-x_{n(k)}, a\right\|+\left\|x_{n(k)-1}-x_{m(k)}, a\right\|\right] \\
& -\phi\left[\left\|x_{m(k)-1}-x_{n(k)}, a\right\|,\left\|x_{n(k)-1}-x_{m(k)}, a\right\|\right] .
\end{aligned}
$$

Also,

$$
\begin{aligned}
\varepsilon \leq & \left\|x_{m(k)}-x_{n(k)}, a\right\| \leq\left\|x_{m(k)}-x_{n(k)-1}, a\right\| \\
& +\left\|x_{n(k)-1}-x_{n(k)}, a\right\| \\
< & \varepsilon+\left\|x_{n(k)-1}-x_{n(k)}, a\right\| .
\end{aligned}
$$

So by using (7), we obtain

$$
\lim _{n \rightarrow \infty}\left\|x_{m(k)}-x_{n(k)}, a\right\|=\varepsilon .
$$

Now

$$
\begin{aligned}
\left\|x_{m(k)}-x_{n(k)-1}, a\right\| \leq & \left\|x_{m(k)}-x_{m(k)-1}, a\right\| \\
& +\left\|x_{m(k)-1}-x_{n(k)}, a\right\| \\
& +\left\|x_{n(k)}-x_{n(k)-1}, a\right\| .
\end{aligned}
$$

Also

$$
\begin{aligned}
\left\|x_{m(k)-1}-x_{n(k)}, a\right\| \leq & \left\|x_{m(k)-1}-x_{m(k)}, a\right\| \\
& +\left\|x_{m(k)}-x_{n(k)}, a\right\| .
\end{aligned}
$$

Therefore,

$$
\lim _{k \rightarrow \infty}\left\|x_{m(k)-1}-x_{n(k)}, a\right\|=\varepsilon .
$$

Similarly, it can be shown that

$$
\lim _{k \rightarrow \infty}\left\|x_{n(k)-1}-x_{m(k)}, a\right\|=\varepsilon .
$$

So from (8), we get

$$
\varepsilon \leq \frac{1}{2}(\varepsilon+\varepsilon)-\phi[\varepsilon, \varepsilon]
$$

implying that $\phi[\varepsilon, \varepsilon] \leq 0$, a contradiction. 
Hence $\left\{x_{n}\right\}$ is a Cauchy sequence and therefore $\left\{x_{n}\right\}$ is a convergent in $X$ and let $x_{n} \rightarrow z \in X$ (say) as $n \rightarrow \infty$. Now for any $a \in X$,

$$
\begin{aligned}
\|z-T(z), a\| \leq & \left\|z-x_{n+1}, a\right\|+\left\|x_{n+1}-T(z), a\right\| \\
\leq & \left\|z-x_{n+1}, a\right\|+\left\|T\left(x_{n}\right)-T(z), a\right\| \\
\leq & \left\|z-x_{n+1}, a\right\| \\
& +\frac{1}{2}\left[\left\|z-T\left(x_{n}\right), a\right\|+\left\|x_{n}-T(z), a\right\|\right] \\
& -\phi\left[\left\|z-T\left(x_{n}\right), a\right\|,\left\|x_{n}-T(z), a\right\|\right] .
\end{aligned}
$$

So by the continuity of $\phi$ and taking $n \rightarrow \infty$, we get

$$
\begin{aligned}
\|z-T(z), a\| & \leq \frac{1}{2}[\|z-T(z), a\|-\phi[0,\|z-T(z), a\|]] \\
& \leq \frac{1}{2}\|z-T(z), a\|
\end{aligned}
$$

implying that $z=T(z)$.

We shall now show that the fixed point $z$ is unique. If possible let $z_{1} \in X$ be another fixed point of $T$, then for any $a \in X$

$$
\begin{aligned}
\left\|z-z_{1}, a\right\|= & \left\|T(z)-T\left(z_{1}\right), a\right\| \\
\leq & \frac{1}{2}\left[\left\|z-T\left(z_{1}\right), a\right\|+\left\|z_{1}-T(z), a\right\|\right] \\
& -\phi\left[\left\|z-T\left(z_{1}\right), a\right\|,\left\|z_{1}-T(z), a\right\|\right] \\
\leq & \left\|z-z_{1}, a\right\|-\phi\left[\left\|z-z_{1}, a\right\|,\left\|z-z_{1}, a\right\|\right],
\end{aligned}
$$

which by property of $\phi$ is a contradiction unless $\left\|z-z_{1}, a\right\|=0$ for all $a \in X$ that is $z=z_{1}$.

This completes the proof.

Example 7. Let $X$ be $\mathbb{R}^{3}$ and consider the following 2-norm on $X$ as

$$
\|x, y\|=\left|\operatorname{det}\left(\begin{array}{ccc}
i & j & k \\
x_{1} & x_{2} & x_{3} \\
y_{1} & y_{2} & y_{3}
\end{array}\right)\right|,
$$

where $x=\left(x_{1}, x_{2}, x_{3}\right), y=\left(y_{1}, y_{2}, y_{3}\right) \in X$. Then $(X,\|\cdot \cdot \cdot\|)$ is a 2 -Banach space (see $[8,9])$.

$T:(X,\|\cdot, \cdot\|) \rightarrow(X,\|\cdot, \cdot\|)$ is defined by $T(x, y, z)=$ $(0,0,1)$ for all $(x, y, z) \in X$. Also let $T(0,0,1)=(0,0,1)$.

Now setting

$$
\phi\left(\left(t, t^{\prime}\right)\right)=\frac{1}{2}\left[t+t^{\prime}\right] ; \quad\left(t, t^{\prime}\right) \in[0, \infty) \times[0, \infty) .
$$

$\phi:[0, \infty)^{2} \rightarrow[0, \infty)$ is a continuous mapping such that $\phi\left(\left(t, t^{\prime}\right)\right)=0$ if and only if $t=t^{\prime}=0$. Now $T(x)=$ $T\left(x_{1}, x_{2}, x_{3}\right)=(0,0,1)$ and $T(y)=T\left(y_{1}, y_{2}, y_{3}\right)=(0,0,1)$. Take $a=\left(a_{1}, a_{2}, a_{3}\right) \in X$.
Now

$$
\|T(x)-T(y), a\|=\left|\operatorname{det}\left(\begin{array}{ccc}
i & j & k \\
0 & 0 & 0 \\
a_{1} & a_{2} & a_{3}
\end{array}\right)\right|=0 .
$$

Also,

$$
\begin{aligned}
\|T(x)-T(y), a\| \leq & \frac{1}{2}[\|x-T(y), a\|+\|y-T(x), a\|] \\
& -\phi[\|x-T(y), a\|,\|y-T(x), a\|] .
\end{aligned}
$$

All the conditions of Theorem 6 are satisfied.

Hence $T$ has a unique fixed point $(0,0,1)$ in $\mathbb{R}^{3}$.

Theorem 8. Let $F$ and $G$ be two self-mappings of a 2-Banach space $X$. Suppose that for any $a \in X$,

$$
\begin{aligned}
\|F(x)-G(y), a\| \leq & \frac{1}{2}[\|x-G(y), a\|+\|y-F(x), a\|] \\
& -\phi[\|x-G(y), a\|,\|y-F(x), a\|],
\end{aligned}
$$

where $\phi:[0, \infty)^{2} \rightarrow[0, \infty)$ is a continuous mapping such that $\phi(x, y)=0$ if and only if $x=y=0$. Then $F$ and $G$ have $a$ unique common fixed point in $X$.

Proof. For $x_{0} \in X$, define $\left\{x_{n}\right\}_{n \geq 1}$ by $x_{2 n+1}=F\left(x_{2 n}\right), x_{2 n+2}=$ $G\left(x_{2 n+1}\right)$. So for any $a \in X$,

$$
\begin{aligned}
\left\|x_{2 n+1}-x_{2 n+2}, a\right\|= & \left\|F\left(x_{2 n}\right)-G\left(x_{2 n+1}\right), a\right\| \\
\leq & \frac{1}{2}\left[\left\|x_{2 n}-G\left(x_{2 n+1}\right), a\right\|\right. \\
+ & \left.\left\|x_{2 n+1}-F\left(x_{2 n}\right), a\right\|\right] \\
& -\phi\left[\left\|x_{2 n}-G\left(x_{2 n+1}\right), a\right\|,\right. \\
& \left.\quad\left\|x_{2 n+1}-F\left(x_{2 n}\right), a\right\|\right] \\
\leq & \frac{1}{2}\left[\left\|x_{2 n}-x_{2 n+2}, a\right\|\right] \\
\leq & \frac{1}{2}\left[\left\|x_{2 n}-x_{2 n+1}, a\right\|\right. \\
& \left.+\left\|x_{2 n+1}-x_{2 n+2}, a\right\|\right] .
\end{aligned}
$$

Hence

$$
\left\|x_{2 n+1}-x_{2 n+2}, a\right\| \leq\left\|x_{2 n}-x_{2 n+1}, a\right\| .
$$

Similarly

$$
\left\|x_{2 n}-x_{2 n+1}, a\right\| \leq\left\|x_{2 n-1}-x_{2 n}, a\right\| .
$$

Thus $\left\{\left\|x_{n}-x_{n+1}, a\right\|\right\}$ is a monotone decreasing sequence of real numbers and hence is convergent and

Let

$$
\lim _{n \rightarrow \infty}\left\|x_{n}-x_{n+1}, a\right\|=r \geq 0 \text { (say) }
$$

We now claim that $r=0$. 
If not,

$$
\begin{aligned}
\left\|x_{2 n+1}-x_{2 n+2}, a\right\| \leq & \frac{1}{2}\left\|x_{2 n}-x_{2 n+2}, a\right\| \\
\leq & \frac{1}{2}\left[\left\|x_{2 n}-x_{2 n+1}, a\right\|\right. \\
& \left.\quad+\left\|x_{2 n+1}-x_{2 n+2}, a\right\|\right] .
\end{aligned}
$$

So,

$$
\lim _{n \rightarrow \infty}\left\|x_{2 n}-x_{2 n+2}, a\right\|=2 r
$$

from (24) taking $n \rightarrow \infty$

$$
r \leq r-\phi[2 r, 0]
$$

implying that $\phi[2 r, 0] \leq 0$, a contradiction.

Therefore, $r=0$.

Now for any integer $p>0$

$$
\begin{aligned}
\left\|x_{n}-x_{n+p}, a\right\| \leq & \left\|x_{n}-x_{n+1}, a\right\|+\left\|x_{n+1}-x_{n+2}, a\right\| \\
& +\cdots+\left\|x_{n+p-1}-x_{n+p}, a\right\| \\
& \longrightarrow 0 \text { as } n \longrightarrow \infty .
\end{aligned}
$$
let

So $\left\{x_{n}\right\}$ is Cauchy in $X$ and hence by completeness of $X$,

$$
\lim _{n \rightarrow \infty} x_{n}=z \in X
$$

Now for all $a \in X$,

$$
\begin{aligned}
\|z-F(z), a\| & \leq\left\|z-x_{n+2}, a\right\|+\left\|x_{2 n+2}-F(z), a\right\| \\
& \leq\left\|z-x_{2 n+2}, a\right\|+\left\|F(z)-G\left(x_{2 n+1}\right), a\right\| .
\end{aligned}
$$

By routine check up, we can see that for sufficiently large values of $n$, Hence, $z=F z$.

Similarly we can show that $z=G(z)$.

We shall now show that $z$ is unique.

If not let $z_{1} \in X$ be another fixed point of $F$ and $G$. Then for any $a \in X$

$$
\begin{aligned}
\left\|z-z_{1}, a\right\|= & \left\|F(z)-G\left(z_{1}\right), a\right\| \\
\leq & \frac{1}{2}\left[\left\|z-z_{1}, a\right\|+\left\|z_{1}-z, a\right\|\right] \\
& -\phi\left[\left\|z-z_{1}, a\right\|,\left\|z_{1}-z, a\right\|\right] .
\end{aligned}
$$

So $\left\|z-z_{1}, a\right\| \leq\left\|z-z_{1}, a\right\|-\phi\left[\left\|z-z_{1}, a\right\|,\left\|z_{1}-z, a\right\|\right]$, showing that $\phi\left[\left\|z-z_{1}, a\right\|,\left\|z_{1}-z, a\right\|\right] \leq 0$. So $z-z_{1}=0$ implies $z=z_{1}$.

Example 9. Let $X$ be $\mathbb{R}^{3}$ and consider the following 2-norm on $X$ as

$$
\|x, y\|=\left|\operatorname{det}\left(\begin{array}{ccc}
i & j & k \\
x_{1} & x_{2} & x_{3} \\
y_{1} & y_{2} & y_{3}
\end{array}\right)\right|,
$$

where $x=\left(x_{1}, x_{2}, x_{3}\right), y=\left(y_{1}, y_{2}, y_{3}\right) \in X$. Then $(X,\|\cdot, \cdot\|)$ is a 2 -Banach space (see $[8,9])$.

Define $F, G: X \rightarrow X$ by $F(x, y, z)=((1 / 4) x$, $(1 / 4) y,(1 / 4) z)$ for all $(x, y, z) \in X$ and $G(x, y, z)=$ $((1 / 3) x,(1 / 3) y,(1 / 3) z)$ for all $(x, y, z) \in X$.

Now setting

$$
\phi\left(\left(t, t^{\prime}\right)\right)=\frac{1}{2} \max \left\{t, t^{\prime}\right\} ; \quad\left(t, t^{\prime}\right) \in[0, \infty) \times[0, \infty) .
$$

$\phi:[0, \infty)^{2} \rightarrow[0, \infty)$ is a continuous mapping such that $\phi\left(\left(t, t^{\prime}\right)\right)=0$ if and only if $t=t^{\prime}=0$. Take $a=\left(a_{1}, a_{2}, a_{3}\right) \in$ $X$.

Now

$$
\begin{aligned}
& \|F(x)-G(y), a\|
\end{aligned}
$$

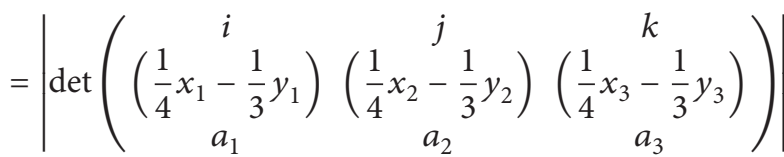

$$
\begin{aligned}
& \|x-G(y), a\|
\end{aligned}
$$

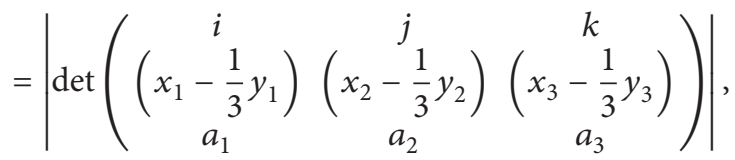

$$
\begin{aligned}
& \|y-F(x), a\|
\end{aligned}
$$

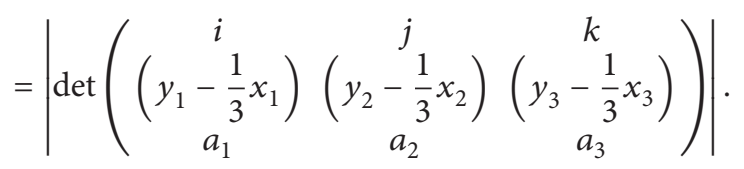

By routine calculation, it can be seen that the condition in Theorem 8

$$
\begin{aligned}
\|F(x)-G(y), a\| \leq & \frac{1}{2}[\|x-G(y), a\|+\|y-F(x), a\|] \\
& -\phi[\|x-G(y), a\|,\|y-F(x), a\|]
\end{aligned}
$$

is satisfied. Also $F$ and $G$ have a unique common fixed point $(0,0,0)$ in $X$.

Theorem 10. Let $(X,\|\cdot, \cdot\|)$ be a 2-Banach space with $T$ : $X \rightarrow X$ and let $\left\{T_{n}: X \rightarrow X\right\}$ be a sequence of mappings such that

(i) for all $u \in X$ and $n=1,2, \ldots$

$$
\begin{array}{r}
\left\|T_{n}(x)-T_{n}(y), u\right\| \leq \frac{1}{2}\left[\left\|x-T_{n}(y), u\right\|\right. \\
\left.+\left\|y-T_{n}(x), u\right\|\right] \\
-\phi\left[\left\|x-T_{n}(y), u\right\|,\right. \\
\left.\left\|y-T_{n}(x), u\right\|\right],
\end{array}
$$


where $\phi:[0, \infty)^{2} \rightarrow[0, \infty)$ is a continuous mapping such that $\phi(x, y)=0$ if and only if $x=y=0$,

(ii) $\lim _{n \rightarrow \infty} T_{n}(x)=T(x)$ for each $x \in X$. Then $T$ has a unique fixed point $z$ in $X$ such that $z=\lim _{n \rightarrow \infty} z_{n}$ where $z_{n}$ is the fixed point of $T_{n}(n=1,2, \ldots)$.

Proof.

$$
\begin{aligned}
& \|T(x)-T(y), u\| \\
& \leq\left\|T(x)-T_{n}(x), u\right\|+\frac{1}{2}\left[\left\|x-T_{n}(y), u\right\|\right. \\
& \quad+\left\|y-T_{n}(x), u\right\| \\
& \quad-\phi\left[\left\|x-T_{n}(y), u\right\|,\left\|y-T_{n}(x), u\right\|\right] \\
& \quad+\left\|T(y)-T_{n}(y), u\right\| .
\end{aligned}
$$

Now

$$
\begin{aligned}
\left\|x-T_{n}(y), u\right\| \leq & \|x-T(y), u\| \\
& +\left\|T_{n}(y)-T(y), u\right\| \\
& \longrightarrow\|x-T(y), u\| \quad \text { as } n \longrightarrow \infty .
\end{aligned}
$$

Similarly

$$
\left\|y-T_{n}(x), u\right\| \longrightarrow\|y-T(x), u\| \quad \text { as } n \longrightarrow \infty .
$$

As $\phi$ is continuous,

$$
\begin{aligned}
& \phi\left[\left\|x-T_{n}(y), u\right\|,\left\|y-T_{n}(x), u\right\|\right] \\
& \quad \longrightarrow \phi[\|x-T(y), u\|,\|y-T(x), u\|] .
\end{aligned}
$$

So from (40) taking limit as $n \rightarrow \infty$, we obtain

$$
\begin{aligned}
\|T(x)-T(y), u\| \leq & \frac{1}{2}[\|x-T(y), u\|+\|y-T(x), u\|] \\
& -\phi[\|x-T(y), u\|,\|y-T(x), u\|] .
\end{aligned}
$$

Hence by Theorem 6, $T$ has a unique fixed point $z$ in $X$.

If possible, let

$$
\lim _{n \rightarrow \infty} z_{n} \neq z \in X
$$

Now for $u \in X$ let

$$
\begin{gathered}
\lim _{n \rightarrow \infty}\left\|z_{n}-z, u\right\|=\alpha>0 \\
\left\|z-z_{n}, u\right\|=\left\|T(z)-T_{n}\left(z_{n}\right), u\right\| \\
\leq\left\|T(z)-T_{n}(z), u\right\|+\left\|T_{n}(z)-T_{n}\left(z_{n}\right), u\right\| .
\end{gathered}
$$

Now

$$
\begin{array}{r}
\left\|T_{n}(z)-T_{n}\left(z_{n}\right), u\right\| \leq \frac{1}{2}\left[\left\|z-T_{n}\left(z_{n}\right), u\right\|\right. \\
\left.+\left\|z_{n}-T_{n}(z), u\right\|\right] \\
-\phi\left[\left\|z-T_{n}\left(z_{n}\right), u\right\|,\right. \\
\left.\left\|z_{n}-T_{n}(z), u\right\|\right] .
\end{array}
$$

Now

$$
\begin{aligned}
\left\|z_{n}-T_{n}(z), u\right\| \leq & \left\|z_{n}-z, u\right\|+\|z-T(z), u\| \\
& +\left\|T(z)-T_{n}(z), u\right\| \\
= & \left\|z_{n}-z, u\right\|+\left\|T(z)-T_{n}(z), u\right\| .
\end{aligned}
$$

So from (46), (47), (48), and (49), we get

$$
\begin{aligned}
\lim _{n \rightarrow \infty}\left\|z-z_{n}, u\right\| \leq & \lim _{n \rightarrow \infty}\left\|T_{n}(z)-T_{n}\left(z_{n}\right), u\right\| \\
\leq & \lim _{n \rightarrow \infty} \frac{1}{2}\left[\left\|z-z_{n}, u\right\|+\left\|z_{n}-z, u\right\|\right] \\
& -\lim _{n \rightarrow \infty} \phi\left[\left\|z-z_{n}, u\right\|,\left\|z_{n}-z, u\right\|\right]
\end{aligned}
$$

Hence, $\alpha \leq(1 / 2)(2 \alpha)-\phi[\alpha, \alpha]$ showing that $\phi[\alpha, \alpha] \leq 0$ which is possible only when $\alpha=0$ $z$.

Hence $\lim _{n \rightarrow \infty}\left\|z-z_{n}, u\right\|=0$, implying that $\lim _{n \rightarrow \infty} z_{n}=$

Theorem 11. Let $X$ be a 2-Banach space, $\left\{T_{n}\right\}$ a sequence of mappings of $X$ onto itself with fixed points $z_{n}$. Let $T$ be a mapping of $X$ into itself satisfying the condition of Theorem 6 with fixed point $z$, such that $T_{n} \rightarrow$ T uniformly on $\left\{z_{n}: n=\right.$ $1,2, \ldots\}$. Then $\lim _{n \rightarrow \infty} z_{n}=z \in X$.

Proof. Fix $\varepsilon>0$. From the uniform convergence of $\left\{T_{n}\right\}$ on $\left\{z_{n}: n=1,2, \ldots\right\}$, there exists an integer $N$ such that for all $n \geq N$, and for all $u \in X,\left\|T\left(z_{n}\right)-T_{n}\left(z_{n}\right), u\right\|<\varepsilon$ for all $z_{n}$.

Now

$$
\begin{aligned}
\left\|z-z_{n}, u\right\| & =\left\|T(z)-T_{n}\left(z_{n}\right), u\right\| \\
& \leq\left\|T(z)-T\left(z_{n}\right), u\right\|+\left\|T\left(z_{n}\right)-T_{n}\left(z_{n}\right), u\right\| .
\end{aligned}
$$

If possible let $\lim _{n \rightarrow \infty} z_{n} \neq z \in X$ that is there exists $u \in X$,

$$
\lim _{n \rightarrow \infty}\left\|z_{n}-z, u\right\|=\alpha>0
$$

Now for all $u \in X$,

$$
\begin{aligned}
\left\|T(z)-T\left(z_{n}\right), u\right\| \leq & \frac{1}{2}\left[\left\|z-T\left(z_{n}\right), u\right\|\left\|z_{n}-T(z), u\right\|\right] \\
& -\phi\left[\left\|z-T\left(z_{n}\right), u\right\|,\left\|z_{n}-T(z), u\right\|\right] .
\end{aligned}
$$

So for $u \in X$ satisfying (52)

$$
\left\|z-T\left(z_{n}\right), u\right\| \leq\left\|z-z_{n}, u\right\|+\left\|T_{n}\left(z_{n}\right)-T\left(z_{n}\right), u\right\| .
$$

Therefore

$$
\lim _{n \rightarrow \infty}\left\|z-T\left(z_{n}\right), u\right\| \leq \varepsilon+\lim _{n \rightarrow \infty}\left\|z-z_{n}, u\right\|=\varepsilon+\alpha .
$$

As $\varepsilon>0$ is arbitrary,

$$
\lim _{n \rightarrow \infty}\left\|z-T\left(z_{n}\right), u\right\| \leq \alpha .
$$


Again

$$
\left\|z-z_{n}, u\right\| \leq\left\|z-T\left(z_{n}\right), u\right\|+\left\|T\left(z_{n}\right)-T_{n}\left(z_{n}\right), u\right\| .
$$

Hence

$$
\lim _{n \rightarrow \infty}\left\|z-z_{n}, u\right\| \leq \lim _{n \rightarrow \infty}\left\|z-T\left(z_{n}\right), u\right\|+\varepsilon
$$

As $\varepsilon>0$ is arbitrary,

$$
\alpha \leq \lim _{n \rightarrow \infty}\left\|z-T\left(z_{n}\right), u\right\| .
$$

Therefore

$$
\lim _{n \rightarrow \infty}\left\|z-T\left(z_{n}\right), u\right\|=\alpha
$$

Hence

$$
\lim _{n \rightarrow \infty} \phi\left[\left\|z-T\left(z_{n}\right), u\right\|,\left\|z_{n}-T(z), u\right\|\right] \longrightarrow \phi[\alpha, \alpha]
$$

Taking $n \rightarrow \infty$ in (53), we get $\alpha \leq(1 / 2)(2 \alpha)-\phi[\alpha, \alpha]$, showing that $\phi[\alpha, \alpha] \leq 0$ which possible only when $\alpha=0$, a contradiction to (52).

Hence $\lim _{n \rightarrow \infty}\left\|z_{n}-z, u\right\|=0$, implying that $\lim _{n \rightarrow \infty} z_{n}=$

Theorem 12. Let $(X,\|\cdot, \cdot\|)$ be a 2-Banach space and for any $x \in X,\left\{T_{n}(x)\right\}_{n=1,2, \ldots}$ a sequence of mappings of $X$ onto itself. Suppose there exists a sequence of nonnegative integers $\left\{m_{n}\right\}$ such that for all $x, y, u \in X$, and every pair $i, j$ with $i \neq j$ satisfying

$$
\begin{array}{r}
\left\|T_{i}^{m_{i}}(x)-T_{j}^{m_{j}}(y), u\right\| \leq \frac{1}{2}\left[\left\|x-T_{j}^{m_{j}}(y), u\right\|\right. \\
\left.+\left\|y-T_{i}^{m_{i}}(x), u\right\|\right] \\
-\phi\left[\left\|x-T_{j}^{m_{j}}(y), u\right\|,\right. \\
\left.\left\|y-T_{i}^{m_{i}}(x), u\right\|\right],
\end{array}
$$

where $\phi:[0, \infty)^{2} \rightarrow[0, \infty)$ is a continuous mappings such that $\phi(x, y)=0$ if and only if $x=y=0$. Then $\left\{T_{n}\right\}$ have a unique common fixed point in $X$.

Proof. Define $S_{i}=T_{i}^{m_{i}}, i=1,2, \ldots$

Then

$$
\begin{array}{r}
\left\|S_{i}(x)-S_{j}(y), u\right\| \leq \frac{1}{2}\left[\left\|x-S_{j}(y), u\right\|\right. \\
\left.+\left\|y-S_{i}(x), u\right\|\right] \\
-\phi\left[\left\|x-S_{j}(y), u\right\|,\right. \\
\left.\left\|y-S_{i}(x), u\right\|\right] .
\end{array}
$$

Pick $x_{0} \in X$ and define $x_{n}=S_{n}\left(x_{n-1}\right), n=1,2, \ldots$
Now

$$
\begin{aligned}
\left\|x_{n}-x_{n+1}, u\right\|= & \left\|S_{n}\left(x_{n-1}\right)-S_{n+1}\left(x_{n}\right), u\right\| \\
\leq & \frac{1}{2}\left[\left\|x_{n-1}-S_{n+1}\left(x_{n}\right), u\right\|\right. \\
& \left.+\left\|x_{n}-S_{n}\left(x_{n-1}\right), u\right\|\right] \\
- & \phi\left[\left\|x_{n-1}-S_{n+1}\left(x_{n}\right), u\right\|,\right. \\
& \left.\left\|x_{n}-S_{n}\left(x_{n-1}\right), u\right\|\right] .
\end{aligned}
$$

By routine calculation, we can see that

$$
\left\|x_{n}-x_{n+1}, u\right\| \leq\left\|x_{n-1}-x_{n}, u\right\| .
$$

Implying that $\left\{\left\|x_{n}-x_{n+1}, u\right\|\right\}$ is a monotone decreasing sequence of real numbers and hence is convergent and let

$$
\lim _{n \rightarrow \infty}\left\|x_{n}-x_{n+1}, u\right\|=r>0
$$

We shall now show that $r=0$.

If not,

$$
\begin{aligned}
\left\|x_{n}-x_{n+1}, u\right\| & \leq \frac{1}{2}\left[\left\|x_{n-1}-x_{n+1}, u\right\|\right] \\
& \leq \frac{1}{2}\left[\left\|x_{n-1}-x_{n}, u\right\|+\left\|x_{n}-x_{n-1}, u\right\|\right]
\end{aligned}
$$

Taking $n \rightarrow \infty$,

$$
\begin{aligned}
r & \leq \frac{1}{2} \lim _{n \rightarrow \infty}\left[\left\|x_{n-1}-x_{n+1}, u\right\|\right] \\
& \leq \frac{1}{2}\left[\lim _{n \rightarrow \infty}\left\|x_{n-1}-x_{n}, u\right\|+\lim _{n \rightarrow \infty}\left\|x_{n}-x_{n+1}, u\right\|\right]=r .
\end{aligned}
$$

Hence

$$
\lim _{n \rightarrow \infty}\left[\left\|x_{n-1}-x_{n+1}, u\right\|\right]=2 r .
$$

Taking $n \rightarrow \infty$ in (64), we get $r \leq r-\phi[2 r, 0]$, showing that $\phi[2 r, 0] \leq 0$ which is possible only when $r=0$.

Now for any integer $p>0$

$$
\begin{gathered}
\left\|x_{n}-x_{n+p}, u\right\| \leq\left\|x_{n}-x_{n+1}, u\right\|+\left\|x_{n+1}-x_{n+2}, u\right\| \\
+\cdots+\left\|x_{n+p-1}-x_{n+p}, u\right\| \\
\longrightarrow 0 \text { as } n \longrightarrow \infty .
\end{gathered}
$$

Therefore $\left\{x_{n}\right\}$ is Cauchy in $X$ and hence by completeness of $X$, let

$$
\lim _{n \rightarrow \infty} x_{n}=z \in X
$$


Now for any integer $m$,

$$
\begin{aligned}
\left\|z-S_{m}(z), u\right\| \leq & \left\|z-x_{n}, u\right\|+\left\|x_{n}-S_{m}(z), u\right\| \\
= & \left\|z-x_{n}, u\right\|+\left\|S_{n}\left(x_{n-1}\right)-S_{m}(z), u\right\| \\
\leq & \left\|z-x_{n}, u\right\|+\frac{1}{2}\left[\left\|x_{n-1}-S_{m}(z), u\right\|\right. \\
& \left.+\left\|z-S_{n}\left(x_{n-1}\right), u\right\|\right] \\
\leq & \left\|z-x_{n}, u\right\|+\frac{1}{2}\left[\left\|x_{n-1}-z, u\right\|\right] \\
& +\frac{1}{2}\left[\left\|z-S_{m}(z), u\right\|\right]+\left\|z-x_{n}, u\right\| .
\end{aligned}
$$

Hence

$$
\begin{aligned}
\frac{1}{2}\left\|z-S_{m}(z), u\right\| \leq & 2\left[\left\|z-x_{n}, u\right\|\right] \\
& +\frac{1}{2}\left[\left\|x_{n-1}-z, u\right\|\right] \\
& \longrightarrow 0 \text { as } n \longrightarrow \infty
\end{aligned}
$$

Therefore $\left\|z-S_{m}(z), u\right\|=0$ implying that $S_{m}(z)=z$ for all $m$.

Hence $\left\{S_{m}\right\}$ has a common fixed point $z$.

If $z_{1} \in X$ is another fixed point of $\left\{S_{n}\right\}$, then

$$
\begin{aligned}
\left\|z-z_{1}, u\right\|= & \left\|S_{n}(z)-S_{n}\left(z_{1}\right), u\right\| \\
\leq & \frac{1}{2}\left[\left\|z-z_{1}, u\right\|+\left\|z-z_{1}, u\right\|\right] \\
& -\phi\left[\left\|z-z_{1}, u\right\|,\left\|z-z_{1}, u\right\|\right] .
\end{aligned}
$$

Implying that $\phi\left[\left\|z-z_{1}, u\right\|,\left\|z-z_{1}, u\right\|\right] \leq 0$. Hence $z-z_{1}=0$ implying that $z=z_{1}$.

Now for each $m$,

$$
T_{n}(z)=T_{n}\left(S_{n}(z)\right)=T_{n}\left(T_{n}^{m_{n}}(z)\right)=T_{n}^{m_{n}}\left(T_{n}(z)\right)
$$

showing that $T_{n}(z)$ is the fixed point of $S_{n}$. for all $n$.

Also $S_{n}(z)=z$ and $S_{n}\left(T_{n}(z)\right)=T_{n}(z)$. Hence $z=T_{n}(z)$

\section{References}

[1] S. Gähler, "2-metric Rume and Ihre Topologische Strucktur," Mathematische Nachrichten, vol. 26, no. 1-4, pp. 115-148, 1963.

[2] K. Iseki, "Fixed point theorems in 2-metric space," Mathematics Seminar Notes, Kobe University, vol. 3, pp. 133-136, 1975.

[3] M. S. Khan and M. D. Khan, "Involutions with fixed points in 2-Banach spaces," International Journal of Mathematics and Mathematical Sciences, vol. 16, no. 3, pp. 429-434, 1993.

[4] M. Saha and D. Dey, "Fixed point of expansive mappings in a 2-Banach space," International Journal of Mathematical Sciences and Engineering Applications, vol. 4, no. 4, pp. 355-362, 2010.

[5] M. Gangopadhyay, M. Saha, and A. P. Baisnab, "Some fixed point theorems for contractive type mappings in 2-Banach space," International Journal of Mathematics and Computer Science, vol. 3, no. 4, pp. 237-246, 2008.
[6] M. Saha and D. Dey, "Some results on fixed points of mappings in a 2-metric space," International Journal of Contemporary Mathematical Sciences, vol. 4, no. 21, pp. 1021-1028, 2009.

[7] B. S. Choudhury, "Unique fixed point theorem for weakly C-contractive mappings," Kathmandu University Journal of Science, Engineering and Technology, vol. 5, no. 1, pp. 6-13, 2009.

[8] M. A. Açikgz, "A review on 2-normed Structures," International Journal of Mathematical Analysis, vol. 1, no. 4, pp. 187-191, 2007.

[9] R. W. Freese and Y. J. Cho, Geometry of Linear 2-Normed Space, Nova Science, New York, NY, USA, 2001. 


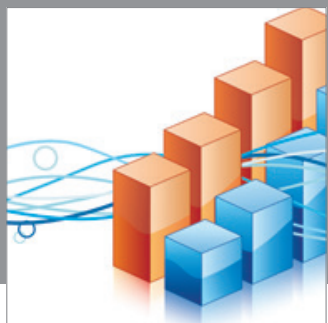

Advances in

Operations Research

mansans

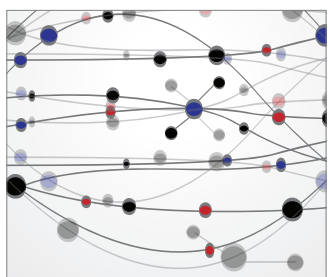

The Scientific World Journal
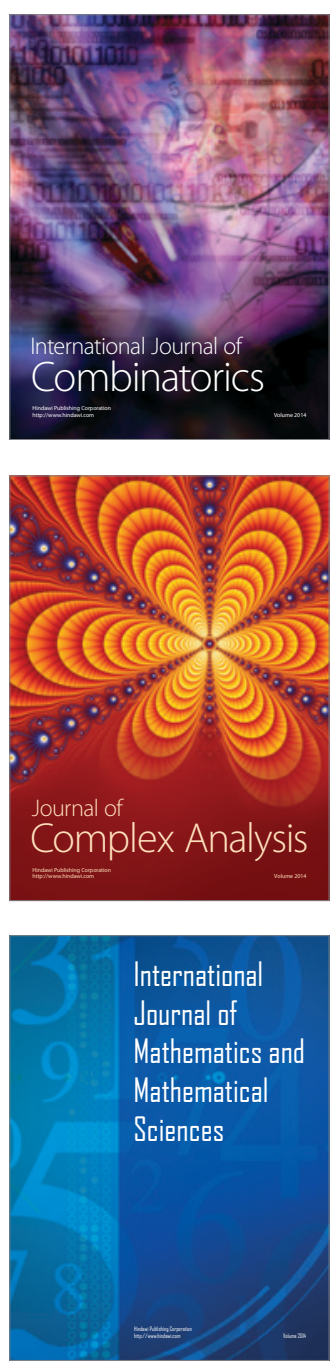
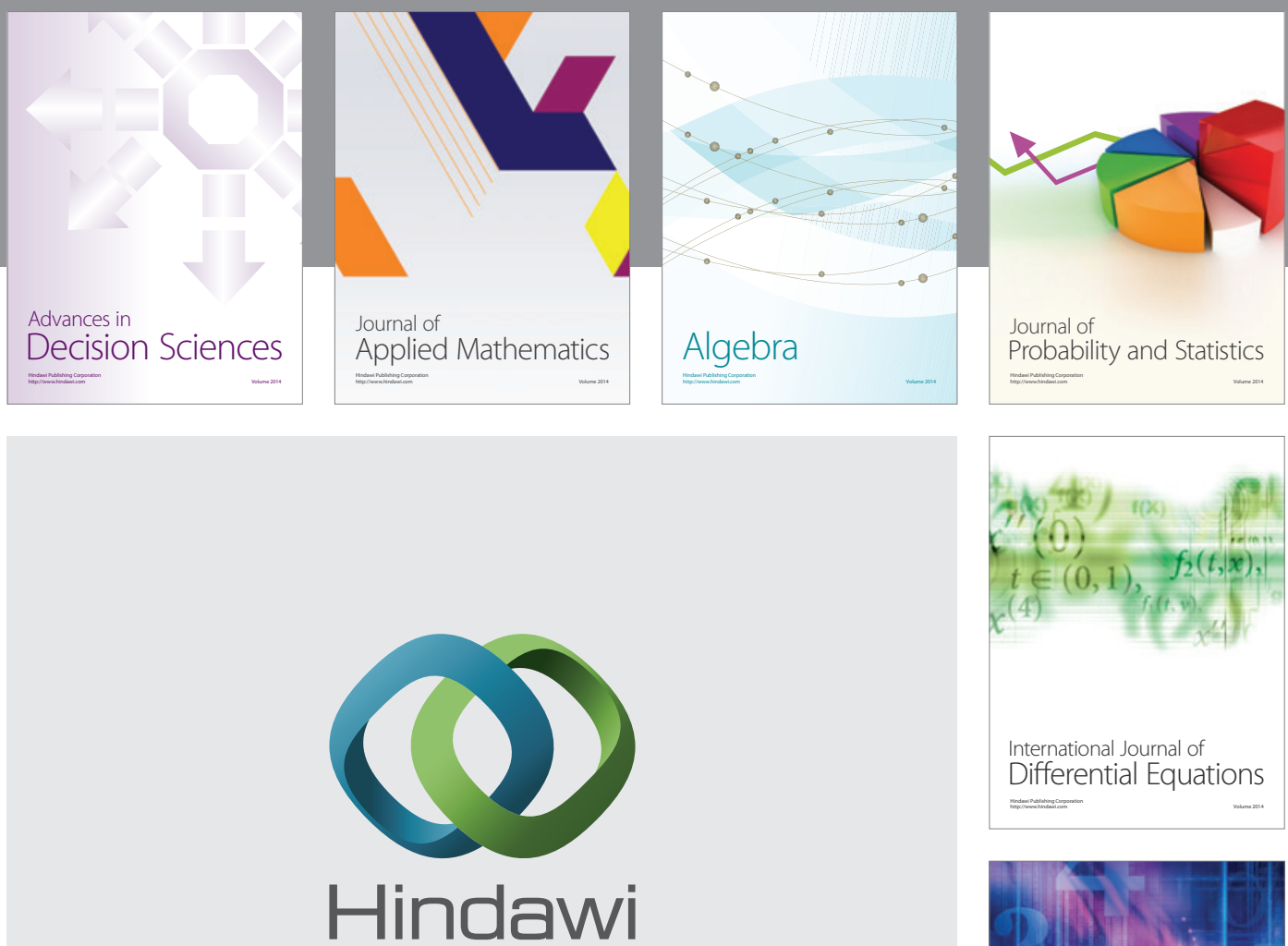

Submit your manuscripts at http://www.hindawi.com
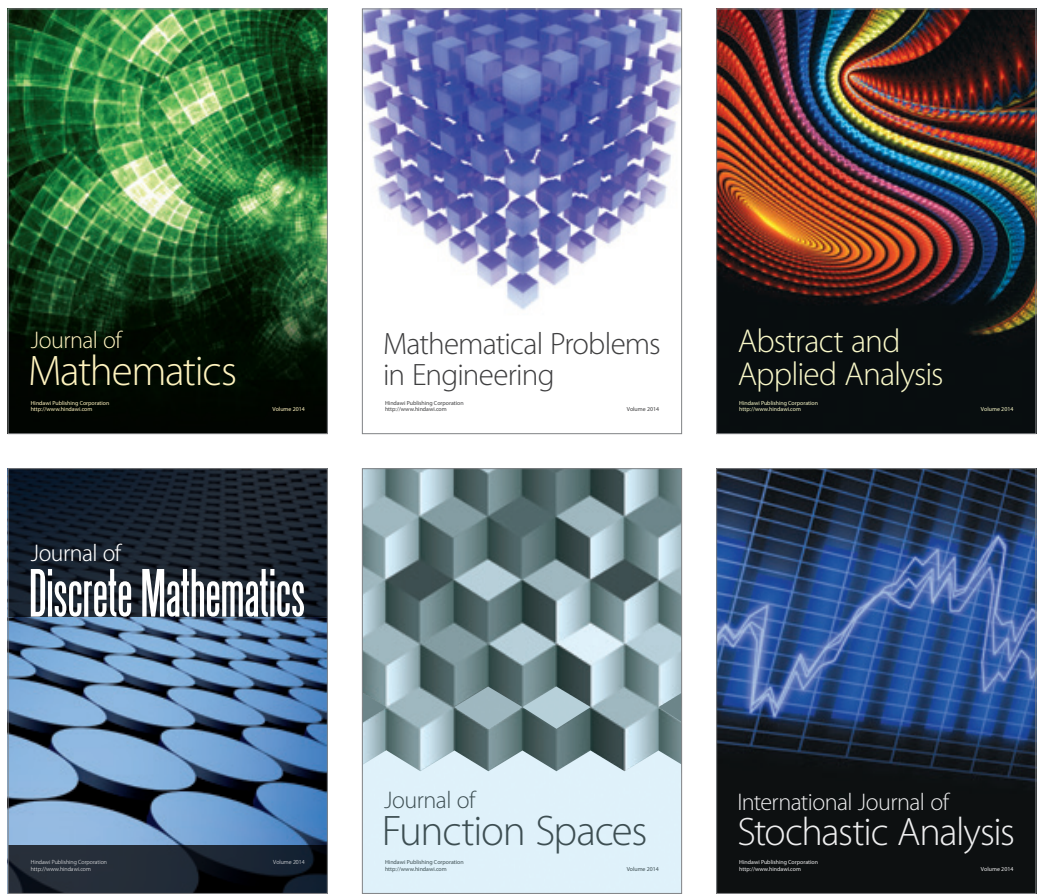

Journal of

Function Spaces

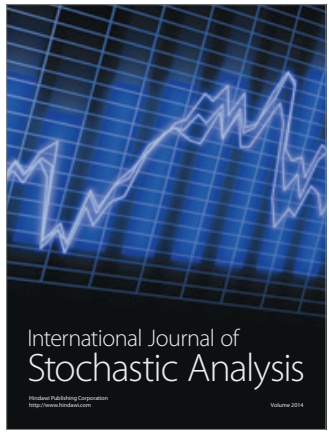

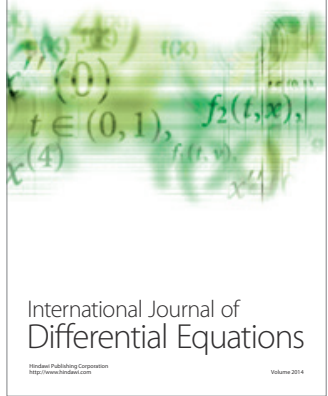
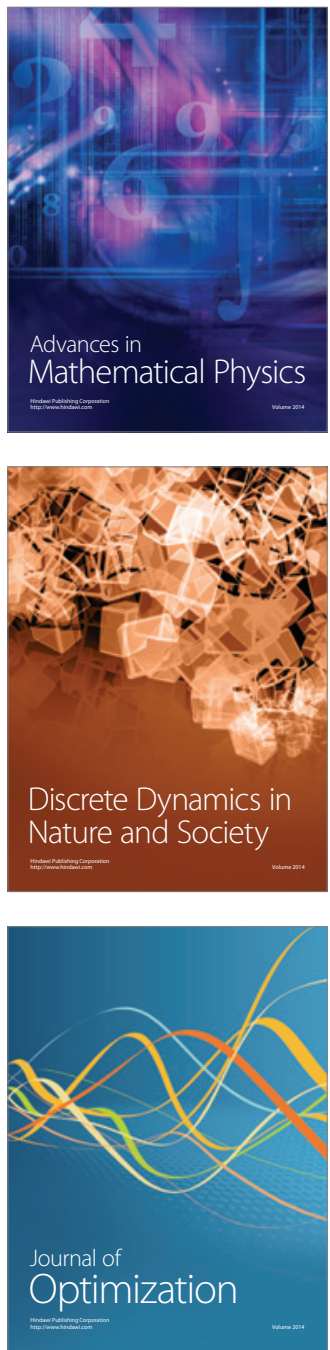